\title{
El espíritu hacker de la libre cultura. Análisis crítico de la tesis de grado de Sergio Yáñez Pérez
}

\author{
The Hacker Spirit of Free Culture. Critical Analysis \\ of the Thesis of Sergio Yáñez Pérez
}

NICOLÁS RIED SOTO

Egresado de Derecho, Chile

RESUMEN Este texto presenta una obra pionera en la problematización del derecho de autor: la tesis de grado del reconocido penalista Sergio Yáñez Pérez (1956). Una obra que cuestiona las bases del derecho de autor sin estar sometida a las condiciones culturales posteriores a la masificación de Internet requiere de nuestra atención, dado que ofrece nuevas perspectivas para el análisis, siendo destacable además que sea una obra chilena y de las primeras de Latinoamérica. El análisis de la obra tendrá como motor los conceptos claves que describen la producción cultural desde el año 2000: el concepto de era de la información de Manuel Castells, el de ética hacker de Pekka Himanen y el de cultura libre de Lawrence Lessig. Esto nos permitirá comprender y enmarcar la discusión sobre derecho de autor en clave política: al pensar el estatus de la producción humana en una comunidad delineamos la forma de esa misma comunidad.

PALABRAS CLAVE Derecho de autor, ética hacker, libre cultura, era de la información. 


\begin{abstract}
This paper presents a pioneering work in the problem of copyright: the thesis of renowned criminalist Sergio Yáñez Pérez (I956). A work that questions the basis of copyright without being subject to postInternet cultural conditions requires our attention because it offers new perspectives for the analysis, being remarkable that it's a Chilean work and one of the firsts in Latin America. The analysis has by motor the keywords that describes the cultural production since 2000: the concept of Age of information by Manuel Castells, Hacker ethic by Pekka Himanen and Free culture by Lawrence Lessig. This will allow us understand and frame the discussion about copyright in political terms: to think the human production status in a community, we outline the shape of this community.
\end{abstract}

KEYWORDS Copyright, hacker ethics, free culture, age of information.

The bad artists imitate, the great artists steal.

Pablo Pieasso Banksy

\title{
INTRODUCCIÓN
}

«En la era de la información...» es la frase con la que presentan su problema una importante cantidad de tesis de grado que abordan el tema del derecho de autor y la propiedad intelectual desde el año 2000. Contextualizar el momento histórico en el que se abordará un problema teórico parece ser fundamental, sobre todo cuando tenemos en cuenta los grandes cambios tecnológicos que afectan la comprensión del objeto de estudio. Sin embargo, parece ser que la llamada era de la información es algo más que el contexto en el cual se desarrollan las tesis sobre derecho de autor, pues parece ser el aparato que constituye la reflexión misma sobre el derecho de autor y la tecnología. La reflexión sobre derecho de autor en Chile se da en los términos asumidos como obvios en un mundo informatizado cuyo paradigma es Internet, lo cual deja de lado ciertas preguntas claves para sentar las bases de la discusión: ¿qué es una obra, comprendida como un objeto cultural y colectivo? ¿Qué es un autor, considerado como participante de una comunidad? ¿Cuál es el modo en que nos relacionamos con la cultura y su producción después de surgida la era de la información? 
Abandonar reflexivamente la obviedad de la era de la información puede servir para dar cuenta de otra perspectiva respecto al problema del derecho de autor y de la cultura libre. Ese abandono podemos hacerlo desde una obra crítica producida en una era preinformática. En Chile la última tesis que habla de derecho de autor sin hacerlo desde la lógica de la era de la información data de I956: la tesis del ilustre jurista don Sergio Yáñez Pérez.

En el año 1956, la tesis La naturaleza jurídica del derecho de autor de Yáñez era una obra de resistencia académica, en el sentido en que constituía en sí misma una ruptura de la disciplina jurídica. Según su revisor, «no hace referencia a ley alguna». La disciplina jurídica que promovía la Facultad de Derecho de la Universidad de Chile en esa época se veía fracturada por esta obra, pues reconociendo su genialidad debía presentarse como un escape al canon jurídico. Lo notable consiste en que es una reflexión filosófica sobre los fundamentos del derecho de autor en una época en que parecía no ser problemático, por lo cual la obra era considerada de mera reflexión, es decir, sin utilidad práctica. Una tesis que hablaba de un problema de otra época, sin mucha posibilidad de ser clasificada como parte de alguna disciplina del derecho y sin tener algún profesor corrector experto en la materia, es una tesis que se transforma en una incomodidad. Cabe mencionar que el autor de la tesis no se dedicó al derecho de autor, sino al derecho penal, debido a que no podía pensarse el derecho de autor como una taxonomía jurídica importante, de modo tal que alguien pudiera dedicar su vida a ello. Nuestro primer dato es que el derecho de autor era una incomodidad.

En I970, en Chile se dictó la Ley I7.336 sobre Propiedad Intelectual. La causa de la publicación se expresa en el mensaje del Presidente:

El actual desarrollo de la técnica en el mundo y el empleo que de ella se hace en la divulgación de las obras artísticas, literarias y científicas, al crear nuevos sistemas y procesos de divulgación, obliga al Estado a revisar las normas vigentes de protección a los creadores intelectuales y subsanar las lagunas de que adolecen.

El advenimiento de la televisión, de las cintas magnetofónicas y de la comunicación por satélites artificiales, crea problemas nuevos que el legislador no puede ignorar, abandonando a la buena o mala fe de los usuarios la utilización del producto del ingenio de los creadores intelectuales. 
Nuestro país no puede mantenerse al margen de los avances legislativos en materia de derechos intelectuales originados en las convenciones internacionales sobre la materia y, muy particularmente, en la Convención Universal sobre Derecho de Autor de I952, la Convención de Roma sobre Derechos Conexos de 196r y la revisión de la Convención Internacional de la Unión de Berna de I967. ${ }^{1}$

Existía ya un desarrollo jurídico de los derechos que amparan a los artistas y sus obras, ligado fuertemente al desarrollo tecnológico de la producción misma del arte, la literatura y la ciencia. Si bien el derecho de autor es una rama del derecho que forma parte del derecho privado, no deja de tener un contenido público en relación con los mecanismos de producción, reproducción y publicación de las obras. Pero de todas maneras la autonomía del derecho de autor se presenta por una cuestión principalmente cultural: el lenguaje y los dilemas con que el derecho tradicional aborda los problemas entre particulares, entre artistas y consumidores, es anquilosado y anacrónico, no permite dar cuenta del mundo nuevo que llegó gracias a la tecnología ni de los avances tecnológicos, pues la analogía no se permite: nada es comparable en el derecho romano con la representación de una obra cinematográfica en formato Blu-ray, nada lo es con la reproducción industrial de una obra literaria en formato PDF, nada lo es con el plagio de una representación fotográfica digital.

Desde el año 2000, el problema del derecho de autor aparece en su autonomía en el marco de la academia. Se vuelve relevante desde un punto de vista sociológico, pues se articula una concepción intelectual que presenta los procesos históricos, sociales y políticos teniendo en cuenta el desarrollo tecnológico producido desde la masificación de Internet. La cuestión informática se vuelve fundamental, en el sentido en que ya no se trata que la analogía no tenga cabida en la aplicación del derecho, que no se pueda comparar un caso tecnológico con otro caso clásico que incluya animales o predios, sino que el mundo comienza un proceso de abstracción en que el caso básico del conflicto entre particulares es de naturaleza abstracta. El derecho ya no depende de su aplicación, sino de su posibilidad de abstracción, pero ¿qué significa esto?

Parece ser que los contextos históricos mundiales son distintos en

I. Biblioteca del Congreso Nacional, Historia de la Ley I7.336, p. 4 . 
cada uno de los hitos mencionados. ¿Qué es lo que hay en el año 2000, que no hay en I956? Si vamos al desarrollo teórico del problema, debemos decir que en el año 2000 hay «era de la información». Es relevante rastrear el desarrollo teórico particularmente influyente de esta expresión en sus raíces filosóficas: de dónde viene y cuál es su repercusión en el debate sobre derecho de autor. En ese sentido, será relevante analizar la influencia de la obra de Yáñez como una obra precursora y resistente, además de darle un rol en el debate actual, respondiendo así a las condiciones de la propia resurrección de la obra. Pero, ¿por qué hacer renacer esta obra en el contexto del debate actual? Si cedemos el punto que la época en la que estaría siendo resucitada la obra de Yáñez es la de la era de la información, uno debería poder reconocer que en la obra misma, publicada en I956, hay un germen de esta era. Para sostener ello diremos que el espíritu de estos tiempos está contenido en la obra de Yáñez, pero dado su desarrollo en otro tiempo nos otorga una perspectiva distinta de análisis, al recordarnos los problemas básicos de la discusión sobre la autoría, la obra y su relación con el derecho.

Para trazar un esquema de lo que son los tiempos que dan forma a la actual discusión sobre derecho de autor, debemos rastrear la discusión teórica que lo contiene. Los conceptos de era de la información e informacionalismo son acuñados por Manuel Castells en su obra publicada en I999, La era de la información. El desarrollo de un espíritu de esos tiempos es generado, desde la misma línea investigativa, por el pensador finlandés Pekka Himanen y su concepto de ética hacker, por lo cual cuando hablamos de "espíritu» hacemos referencia al ethos de los tiempos que caracterizan los modos de producción en el informacionalismo. Lo hacker, en este sentido, se refiere a un modo especial de producción de conocimiento que surge en la disciplina informática, pero que se desarrolla en todos los ámbitos de la cultura sin quedar restringido a ella, siendo su gran antecedente el modo en que la academia reproduce su conocimiento. De esta manera, revisaremos cuál es el lugar de la obra de Yáñez en la era de la información y, junto con ello, tendremos en cuenta el desarrollo particular en el cual el derecho de autor configura su propia naturaleza.

No sólo es relevante, en este sentido, el contexto mundial de la era de la información, el espíritu de estos tiempos, sino además la manera en que la producción de cultura ha cambiado en las últimas décadas, para 
lo cual otra publicación es de particular relevancia: Cultura libre (2004), de Lawrence Lessig. En el análisis de Lessig, la cultura del ciberespacio producida por Internet comienza de la premisa de que los problemas de derecho de autor en el marco de la organización de la producción cultural contemporánea no se acaban en la dimensión propia de Internet, sino que la trascienden y se convierten en problemas AFK. ${ }^{2}$ La producción cultural abandona de facto el paradigma del "permiso", para convertirse por necesidad en uno de acceso libre, en particular debido a la imposibilidad de sancionar normativamente las relaciones virtuales. Las legislaciones relativas a derecho de autor y acceso a la cultura no responden a los verdaderos modos de producción de la cultura, los cuales de hecho son libres; no responden al espíritu que determina esa producción; tampoco responden al proceso histórico en que la producción cultural se halla inmersa.

Reforzando: ¿cuál es el sentido de retraer la obra de Yáñez a este complejo contexto? En la era de la información (Castells), caracterizada por un modo libre de producción cultural (Lessig) y desarrollada en un modo hacker (Himanen), la obra de Yáñez nos ofrece una perspectiva no cargada de estas discusiones, lo cual es valioso dado que nos permite reflexionar desde los cimientos de la discusión y rescatar nuevas preguntas para iluminar viejos problemas. Además, debemos destacar que la obra de Yáñez marca un hito como la primera obra que destaca a nivel nacional en el debate sobre la naturaleza del derecho de autor: cualquier obra actual debería tener en cuenta esta obra. Finalmente, el mérito es que constituye una reflexión filosófica; el demérito que los evaluadores encontraron en la tesis de Yáñez debe ser el brillo que nosotros logremos pulir.

2. «AFK» es una sigla de la jerga hacker para señalar «away from keyboard» («lejos del teclado»). En el documental TPB AFK: The Pirate Bay Away From Keyboard (Simon Klose, 20I3), que muestra el juicio realizado en Suecia a los miembros fundadores del motor de búsqueda y tracker The Pirate Bay, hay una escena en que la abogada de la parte acusante les pregunta desde hace cuánto se conocen «IRL» («in real life», «en la vida real»), a lo que ellos responden: "No creemos en la sigla $I R L$, creemos en la sigla $A F K$. Creemos que Internet es la realidad». 


\section{EL ESPÍRITU HACKER EN LA ERA DE LA INFORMACIÓN}

Manuel Castells introduce el concepto de era de la información en el campo de la sociología a finales del siglo pasado, siendo ya en el año 2000 una obra de alta influencia en la discusión. Conceptos como «globalización», «sociedad red» y «aldea global» forman parte del campo semántico que articula la discusión sobre el estado del mundo tras la caída del socialismo y la articulación del liberalismo como ideología hegemónica. El paradigma informacionalista no tiene necesariamente como punto central de explicación el hecho de que la información sea la principal fuente de poder. Se trata de algo más específico.

Basándose en la noción de paradigma introducida en el debate académico por Thomas S. Kuhn en su importante obra La estructura de las revoluciones científicas (I962), Castells analiza la sociedad red y el informacionalismo respecto a su relación con la tecnología:

El tipo de tecnología que se desarrolla y difunde en una determinada sociedad modela decisivamente su estructura material. Los sistemas tecnológicos evolucionan de forma gradual hasta que se produce un cambio cualitativo de primera magnitud: una revolución tecnológica, que lleva a un nuevo paradigma tecnológico (Castells, 2002: IIO).

Los cambios culturales que conlleva un cambio tecnológico son percibidos, dice Castells, según rendimientos de acumulación de riqueza y poder. Estos son los tiempos del informacionalismo, lo que no quiere decir que la información sea particularmente determinante en términos de acumulación de riqueza y poder, porque eso siempre ha sido así. Lo relevante en términos del informacionalismo para Castells consiste en que:

El informacionalismo es un paradigma tecnológico. Concierne a la tecnología, no a la organización social ni a las instituciones. El informacionalismo proporciona la base para un determinado tipo de estructura social que denomino la 'sociedad red'. Sin el informacionalismo, la sociedad red no podría existir, pero esta nueva estructura social no es producto del informacionalismo, sino de un patrón más amplio de evolución social [...] Lo distintivo de nuestra época histórica es un nuevo paradigma tecnológico marcado por la revolución en la tecnología de la información, y centrado en torno a un racimo de tecnologías informáticas. Lo nuevo es la tecnología del procesamiento de la información 
y el impacto de esta tecnología en la generación y aplicación del conocimiento. Por esta razón no me sirvo de las nociones de economía del conocimiento o sociedad de la información, y prefiero el concepto de informacionalismo: un paradigma tecnológico que se basa en el aumento de la capacidad humana de procesamiento de la información en torno a las revoluciones parejas en microelectrónica e ingeniería genética (Castells, 2002: II I-II2).

Castells considera que la microelectrónica y la ingeniería genética contienen un esquema de producción tecnológica basada en la información y su capacidad autopoiética, recombinatoria y distributiva, que sirve de fundamento para la sociedad red, junto con el sistema económico capitalista y los valores políticos de la libertad. La producción tecnológica está enfocada hacia la producción y almacenamiento de información: la información es lo más relevante, porque cambia y da sentido a nuestros modos de vivir. Este cambio tecnológico, económico y político que trae la instauración del paradigma informacionalista implicó históricamente un cambio ético en cuanto a los modos de producción.

Es Pekka Himanen quien presenta la idea de ética hacker en el marco de la era de la información y desde una particular lectura del clásico texto de Max Weber La ética protestante y el espíritu del capitalismo. La ética hacker es el espíritu de los tiempos descritos por Castells. El desarrollo tecnológico ha configurado un modo de producción de cultura y de distribución del conocimiento particular, basado en la abstracción, es decir, en la inexistencia de rivalidad de bienes y de imposibilidad de daño moral inherente en la propiedad. ${ }^{3}$ Ante estas características de la producción, la discusión jurídica se ha centrado en cómo reconducir la cuestión a las obligaciones y responsabilidades del uso, como si de propiedad se tratara, propiedad en su versión «intelectual», siendo así de particular interés ciertas discusiones: la originalidad subjetiva y objetiva de la obra, los límites de la protección de los derechos del autor, los equilibrios entre la regulación y la permisión, la piratería, etcétera. ${ }^{4}$ Estas discusiones, sin

3. Estas tesis son desarrolladas de gran manera en Wilson (2008). Para el concepto de «abstracción», véase Wark (2006).

4. Para el problema de la originalidad de la obra, véase Real Márquez (200I) y contrastar con Raffo (20II). Para la discusión chilena sobre equilibrios regulatorios, véase Álvarez Valenzuela (20II). 
embargo, están inmersas en una determinada manera de comprender el problema del derecho de autor y la tecnología: la lógica según la cual la producción de conocimiento está ligada a la producción económica. Un quiebre de esa lógica es lo que caracteriza a la ética hacker según Himanen, no la ética del trabajo, no la ética del enriquecimiento, sino una ética caracterizada por la pasión: «Se trata de un espíritu general: los hackers programan porque para ellos los desafíos de la programación tienen un interés intrínseco. Los problemas relacionados con la programación despiertan una curiosidad genuina en el hacker y suscitan su deseo de continuar aprendiendo» (Himanen, 2002: I4). Como mencionábamos, si bien esta forma de comprender y de vivir la relación entre producción y conocimiento tiene su representación ejemplar en la comunidad hacker de la informática, su antecedente más directo es la producción de las comunidades académicas: en la producción de las comunidades académicas es donde podemos encontrar publicación libre y gratuita, circulación de conocimiento sin permisos, uso correcto de fuentes y citas, en definitiva una forma de vida que se adecua no al modo de producción capitalista sino al de la cultura libre, todo porque la finalidad última no es el lucro o la producción material, sino la producción de más conocimiento y cultura.

En la lectura de Max Weber, el espíritu del capitalismo tiene su principal antecedente en el monasterio; según Pekka Himanen, el espíritu hacker lo tiene en la academia. La diferencia fundamental entre los dos modos de producción es el tiempo. En el monasterio, era fundamental tener una noción del tiempo y de la disciplina, comprender que el «tiempo es oro» y que sólo el trabajo nos permitirá la salvación, que el trabajo es un castigo; en la academia, y en particular en la era de la información, lo relevante es el producto, el conocimiento y su divulgación, su discusión y la creación de comunidad, por lo cual el tiempo no adquiere una función disciplinar, sino una libertad: "Desde un punto de vista histórico, esta libertad para autoorganizar el tiempo encuentra de nuevo un precursor en la academia. La academia siempre defendió la libertad de la persona para organizar por sí misma el tiempo» (Himanen, 2002: 33 ).

Así, tanto Castells como Himanen tienen en mente una pregunta por el lugar de la producción en nuestra comunidad: Castells otorga un sentido, que es la producción de información; mientras Himanen otorga un modo: la producción de esa información debe ser hacker, es decir, que debe fundarse principalmente en la libertad. 


\section{LA CULTURA LIBRE Y EL DERECHO DE AUTOR}

Podemos pensar la obra del mismo Yáñez como un robo. No produjo algo nuevo, sino que compiló y tradujo ideas escritas en otros idiomas, articulando una teoría sobre la naturaleza jurídica del derecho de autor. Sin embargo, pensar de ese modo es no comprender que toda forma de presentación es ya una nueva manera de articular un discurso, es constituir una nueva obra. Una republicación de la tesis de Yáñez no puede ser comparada con la publicación primera: en el marco de una tesis para optar al grado en ciencias jurídicas y sociales, en el contexto de una disciplina jurídica estricta, tras haber pasado sólo una década desde una guerra mundial, sin la posibilidad de pensarse la influencia autoral de algo como Internet. El texto es el mismo, pero su lectura no puede ser la misma.

Tenemos entre 1956 y 2000 un período de transformación de la sociedad, un período en que los modos de producción y acceso cultural son otros, el paradigma es otro. En este sentido, es relevante un caso anecdótico: Castells funda su teoría de la sociedad de la información en un concepto clave: el de paradigma, concepto que fue acuñado por Thomas S. Kuhn, por lo que cabe preguntarse si acaso la obra de Castells es absolutamente original o no. Si acaso la obra de Castells es un «robo», tal como lo sería la tesis de Yáñez, es una cuestión que abre la pregunta por la libertad dentro de una cultura democrática que debate las ideas sin personalizarlas y de manera racional: ¿cómo debatir ideas libremente en el foro público si las ideas tienen cadenas y sus dueños pueden ocultarlas? Yáñez dejó de dar por sentado un paradigma, que es el de cierta concepción del derecho de autor, cuestionando el estatus de libre circulación de las obras. El derecho de autor no es un derecho como cualquier otro, es «un derecho sui generis» dice, a lo que podríamos añadir que todo derecho sui generis requiere de una concepción sui generis de la participación en la comunidad.

Otra obra fundamental para comprender la relación del derecho de autor y la comunidad fue, y sigue siendo, el libro Cultura libre, de Lawrence Lessig, publicado en 2004. En este libro, que argumenta a favor de una tradición occidental de la libre cultura que impacta con ciertas prácticas jurídicas que intentan controlar modos no comerciales de producción de cultura, en contra de todo sentido común, se halla la idea 
del cambio de paradigma necesario para que el derecho sea funcional a la comunidad. Mediante una serie de casos ejemplares, Lessig argumenta en contra de la incomprensión de la "piratería» como delito y de la «propiedad» como objeto sacro, teniendo como base la idea de que los avances tecnológicos producen cambios sociales y que estos producen una práctica cultural que, muchas veces, es contradicha por la práctica jurídica. Lo interesante es cómo esa práctica cultural siempre ha estado marcada por un modo de producción cultural en que los productos del intelecto son siempre patrimonio de un espacio común y que las nuevas obras aprovechan de manera positiva toda la base cultural acumulada. Nuestra cultura siempre ha sido libre, en palabras de Lessig:

Una cultura libre apoya y protege a creadores e innovadores. Esto lo hace directamente concediendo derechos de propiedad intelectual. Pero lo hace también indirectamente limitando el alcance de estos derechos, para garantizar que los creadores e innovadores que vengan más tarde sean tan libres como sea posible del control del pasado. Una cultura libre no es una cultura sin propiedad, del mismo modo que el libre mercado no es un mercado en el que todo es libre y gratuito. Lo opuesto a una cultura libre es una 'cultura del permiso' —una cultura en la cual los creadores logran crear solamente con el permiso de los poderosos, o de los creadores del pasado (Lessig, 2004: 4).

Esto no es anarquía, insiste Lessig a lo largo de su obra. Lo que propone es que ciertos aspectos de la producción cultural deben ser regulados por el derecho, incluso aceptando la exótica idea de «propiedad intelectual». Sin embargo, los objetos de control jurídico deben ser aquellos que sean objeto de comercialización, no aquellos que no forman parte de la comercialización. La producción cultural no comerciable es todo el punto: es ella la que constituye el motor del progreso cultural, en un sentido productivo. Lo cual, siguiendo a Lessig, constituye una tradición de nuestra cultura y que ha sido radicalizado por el mundo virtual del Internet: cuando Walt Disney toma los cuentos de los hermanos Grimm, cuando cantamos el Cumpleaños feliz, cuando interpretamos un pasaje de Shakespeare. Lo extraño - y es hacia donde radicalmente quiere dirigirse el derecho en su forma anticultural- es querer controlarlo todo: controlar cuando usemos una escena de una película en una producción cinematográfica, cuando pongamos una canción en nuestro bar, cuando 
veamos un videoclip en un área que no esté permitida su reproducción, cuando descarguemos un archivo de audio que contenga una canción con derechos de propiedad intelectual. Eso es lo raro, pues nuestra cultura es una cultura libre, dice Lessig.

La pregunta difícil de responder es, por tanto, no si una cultura es libre. Todas las culturas son libres en cierto grado. La pregunta, por contra, es ¿̇hasta qué punto es libre nuestra cultura? ¿Cuán libre, y con qué margen, es esta cultura libre para que otros tomen de ella y se basen en ella? ¿Está esa liberad limitada a los miembros del partido? ¿A los miembros de la familia real? ¿A las diez corporaciones en lo alto de la lista de la bolsa de Nueva York? ¿O está esta libertad ampliamente difundida? ¿Para los artistas en general, estén afiliados al MET o no? ¿Para los músicos en general, sean blancos o no? ¿Para los directores de cine en general, estén afiliados a un estudio o no? (Lessig, 2004: 42).

La pregunta de Lessig es relevante en el sentido que adquiere el concepto «libre», como el modo de producción de nuestra cultura y en especial con cómo se concibe en este esquema la idea de derecho de autor, precisamente porque la comprensión de la producción de obras autorales se centra en el concepto de «originalidad», atribuyendo cierta lógica individualista a la creación, que a su vez determina en la elaboración de incoherentes argumentaciones sobre lo original en la obra. Lo interesante de la relación entre la idea de libre cultura y el derecho de autor radica en la comprensión radical de la obra autoral como objeto cultural, y ya no como obra del intelecto.

En su muy lúcido texto Derecho autoral. Hacia un nuevo paradigma, Julio Raffo sostiene que el derecho de autor, tal y como se usa jurídicamente en la actualidad, carece de una reflexión profunda sobre el estatus tanto de la obra como del autor. Sin esa reflexión, el derecho de autor se convierte en un intento desesperado por darle firmeza a una torre de naipes, ya que termina protegiendo a la obra y los derechos que de ella emanan, pero no al autor, en detrimento de la producción cultural. Así, la defensa que realiza Raffo va en el sentido de comprender la obra como un fenómeno cultural:

Podemos decir que toda obra autoral es, básicamente, un fenómeno cultural que tiene siempre una dimensión expresiva y comunicacional de múltiples contenidos posibles porque [...] lo que ella expresa no de- 
pende exclusivamente de lo que ella es, ni de lo que pretendió su autor que ella sea, sino que su sentido surgirá de su integración con su época, con sus circunstancias y con la dimensión cultural y con la sensibilidad de sus destinatarios (Raffo, 20II: 30).

El problema jurídico del derecho de autor tiene como insumo una cuestión cultural, que no puede ser resuelta desde un punto de vista meramente economicista o jurídico, sino que requiere de una comprensión del asunto como una cuestión política en el sentido en que es un problema relativo a la producción cultural en nuestra comunidad, que tiene que ver con sus modos de producción, con los límites de su reproducción y, principalmente, con el patrimonio cultural de la comunidad, esto es, con lo que la comunidad hace, de algún modo, con su identidad. Nadie realiza una obra solo, toda obra es arrojada a la comunidad.

\section{LA NATURALEZA JURÍDICA DEL DERECHO DE AUTOR COMO OBRA CRÍTICA}

El contexto en que la obra de Sergio Yáñez aparece por primera vez ( 1956 ) queda expresado de manera patente en la presentación de su tesis de grado realizada por el profesor guía, Enrique Escala Barros:

No se trata en el presente caso de un estudio de derecho positivo ni hay referencias al derecho nacional o foráneo sobre la materia, como tampoco a las últimas convenciones internacionales que se han ocupado de este tema. El candidato ha encarado, con considerable seriedad científica, un estudio histórico filosófico de la naturaleza del derecho de autor, muchos de cuyos aspectos escapan a la órbita de las materias propias de este seminario.

Podemos delinear, con este extracto, qué era lo que se exigía de una tesis de grado «normal»: el seminario en que participó Yáñez versaba sobre derecho comercial e industrial, materias áridas y estrictamente positivistas en el estudio del derecho, en que una tesis - incluso hoy- sobre fundamentos filosóficos y no contenidos en ninguna ley o doctrina extranjera no sería bienvenida. El positivismo reinante a comienzos de la segunda mitad del siglo XX en la Facultad de Derecho de la Universidad de Chile agudizaba esa disonancia: una tesis que no hiciera referencia alguna a una ley era impensable. Así, a pesar de ser una tesis formidable, es penalizada: nota 6 sobre 7 , precisamente por ser una tesis disidente. 
No sólo disidente en cuanto a la disciplina jurídica, sino en relación con la discusión en la que, de manera incipiente, Yáñez aterriza: «Las obras son susceptibles de reproducción hasta el infinito, el pensamiento se expande de forma irresistible, como las olas de un océano y no reconoce barreras», sostiene en la introducción de su obra.

Yáñez busca desentrañar el fundamento jurídico mediante el cual legitimar la existencia del derecho de autor, para lo cual revisa una serie de argumentaciones que buscan defender ciertas posiciones en lo relativo al derecho que un autor tiene sobre las obras de su creación. La obra se divide en dos partes: en la primera, el autor analiza el estado de la cuestión, las diferentes propuestas para fundamentar la naturaleza del derecho de autor, proponiendo que existen «teorías primarias» de la naturaleza jurídica del derecho de autor, y otras teorías que se presentan como versiones que superan estas teorías primarias; en la segunda sección, propone una sistemática de la naturaleza jurídica del derecho de autor, para lo cual analiza los diversos elementos que constituyen al derecho de autor, y que permitirían darle un espacio dentro de un sistema jurídico en su completitud.

Sobre esta estructura del texto, cabe reflexionar en lo relativo a la necesidad que Yáñez vio al intentar fundamentar filosóficamente la naturaleza jurídica del derecho de autor, sobre todo teniendo en cuenta las condiciones actuales de la discusión: ¿no es, acaso, la discusión jurídica sobre derecho de autor y nuevas tecnologías una cuestión pantanosa precisamente por el escaso desarrollo teórico que presenta? El hecho que desde la discusión jurídica se relegue de modo despectivo la cuestión de los fundamentos al cajón improductivo de «lo filosófico» es precisamente lo que permite mantener un statu quo en una discusión que es de carácter más bien político, pues no sólo hay en discusión cuestiones de derecho privado, sino relevantes problemas de derecho público y derechos fundamentales. Lo importante en la reflexión de Yáñez es precisamente el hecho de articular un margen de discusión desde un núcleo académico jurídico, para extender los márgenes de esa discusión y producir el terreno que permitiría seguir rutas de investigación que demuestren que existe interconexión entre las disputas jurídicas, las políticas y las filosóficas. La labor del crítico es unir lo que parece desunido, juntar lo separado y hacer común lo que parece privatizado.

Esta obra adquiere un valor fundamental como herramienta concep- 
tual e histórica desde el punto de vista de las deficiencias en el trato respecto a la discusión local. La reflexión de Yáñez es jurídica, en el sentido en que el objeto de análisis y problematización se enmarca en el plano del derecho, pero a la vez porque opera bajo una comprensión amplia de lo jurídico entendido como un ámbito cultural: lo que permite a Yáñez presentar su tesis de grado como una tesis de la carrera de derecho es que viene a resolver un problema jurídico con herramientas que provienen de otras esferas. Sin embargo, el problema del derecho de autor, como mostramos más arriba, no se ve limitado por los lindes legales, pues estando inmersa en la era de la información la cuestión cobra sentido como problema cultural, y por tanto sus soluciones se presentan en esa línea: los derechos de autor pueden ser leídos como un problema patrimonial, como un problema moral, pero también como un problema político. Lo que abre Yáñez es, precisamente, un modo de leer la cuestión como un problema político: es necesario hacerse cargo de la naturaleza del derecho de autor en términos jurídicos, porque es un hecho cultural que es propio de los tiempos que el autor veía y prevenía, es un problema de la comunidad y determinar si corresponde al derecho o no es una decisión política, a la que la propia tesis responde de manera afirmativa. El acto más relevante de la propia tesis es ése: afirmar que la cuestión del derecho de autor es un problema jurídico, más allá de las hipótesis de fundamentación al mismo, pues de esta manera Yáñez conecta ámbitos que se creían separados y convierte el problema del derecho de autor en un problema de la comunidad y no sólo de los expertos. Ése es el giro político de una obra como ésta: hacer que un problema que parece de expertos sea un problemas de todos y que sus aristas tienen que ver con cómo pensamos nuestra comunidad, cómo pensamos nuestros modos de producción, cuáles son los límites de nuestra libertad, cuál es el valor que le damos al flujo de ideas, cómo valoramos el hacer humano. Todas esas preguntas no tienen expertos para responderlas, sino que sus respuestas se encuentran precisamente en cómo damos cuenta de la comunidad en que vivimos.

Yáñez permite reconocer que el problema del derecho de autor no es una cuestión que se resuelva simplemente con los instrumentos jurídicos existentes, porque es más que un problema de interpretación de leyes o tratados (por lo cual es coherente que, siendo una tesis jurídica, no cite legislación). Yáñez permite reconocer este problema como un problema 
cultural, relativo a nuestra identidad como comunidad, en definitiva un problema político que viene a dar forma a la cuestión de cómo caracterizamos nuestra participación en la comunidad: hay quienes comprenden que su obra es sólo posible por formar parte de una comunidad, mientras otros confían en que su propio genio y luz produjo todo lo que hacen. El espíritu hacker de la libre cultura implica cierta noción de nosotros en la comunidad que da cuenta que ésta sólo se constituye toda vez que producimos en ella y en su nombre: el que crea sólo es una bestia o un dios, quedando fuera de la comunidad en ambos casos. Hay que pensar la producción cultural en un sentido político, esto es, de manera indisociable con el modo en cómo pensamos nuestra participación en aquello que es común a todos.

\section{REFERENCIAS}

Álvarez Valenzuela, Daniel (20I I). «En busca de equilibrios regulatorios: Chile y las recientes reformas al derecho de autor». International Centre for Trade and Sustainable Development (ICTSD), Documento de Política I 2, Ginebra, Suiza.

Castells, Manuel (2002). «Informacionalismo y sociedad red». En Pekka Himanen, La ética del hacker y el espíritu en la era de la información. Disponible en <http://eprints.rclis.org/I $285 \mathrm{I} / \mathrm{I} /$ pekka.pdf>.

Himanen, Pekka (2002). La ética del hacker y el espíritu en la era de la información. Disponible en <http://eprints.rclis.org/I285I/I/pekka.pdf>.

Lessig, Lawrence (2004). Cultura libre. Disponible en <http://www. worcel.com/archivos/6/Cultura_libre_Lessig.pdf >.

Raffo, Julio (20I I). Derecho autoral. Hacia un nuevo paradigma. Buenos Aires: Marcial Pons.

Real Márquez, Monserrat (200I). «El requisito de la originalidad en los derechos de autor». En Portal Internacional de la Universidad de Alicante sobre Propiedad Industrial e Intelectual y Sociedad de la Información. Disponible en <http://www.uaipit.com/files/publicaciones/000000I974_la\% 20originalidad-art-uaipit2.pdf $>$.

WARK, McKenzie (2006). Un manifiesto hacker. Barcelona: Alpha Decay.

Wilson, James (2008). "Could There Be a Right to Own Intelectual Property?» Law and Philosophy, 28 (4): 393-427. 
YÁÑEz Pérez, Sergio (I956). La naturaleza jurídica del derecho de autor. Tesis de grado. Santiago: Universidad de Chile.

\section{SOBRE EL AUTOR}

Nicolás Ried Soto es egresado de la Facultad Derecho de la Universidad de Chile, coinvestigador Fondecyt y ayudante de las carreras de Derecho y de Teoría del Arte, de la Universidad de Chile. El presente artículo es una versión resumida de su memoria para optar al grado de Licenciado en Ciencias Jurídicas y Sociales. Su correo electrónico es <nicolasried@gmail.com> y su dirección postal Jorge Washington I352, Cerro Navia.

Este trabajo fue recibido el 20 de enero de 20 I 4 y aprobado el 23 de enero de 2015 . 
